\title{
ANÁLISE POR COMPONENTES PRINCIPAIS DE ESPECTROS NEXAFS NA ESPECIAÇÃO DO MOLIBDÊNIO EM CATALISADORES DE HIDROTRATAMENTO
}

\author{
Arnaldo da C. Faro Jr**, Victor de O. Rodrigues e Jean-G. Eon \\ Instituto de Química, Universidade Federal do Rio de Janeiro, Centro de Tecnologia, Bl. A, Cidade Universitária, 21949-900 Rio \\ de Janeiro - RJ, Brasil \\ Angela S. Rocha \\ COPPE - Instituto Alberto Luiz Coimbra de Pós-graduação e Pesquisa de Engenharia, Universidade Federal do Rio de Janeiro, \\ CP 68502, Cidade Universitária, 21949-900 Rio de Janeiro - RJ, Brasil
}

Recebido em 11/11/09; aceito em 9/3/10; publicado na web em 20/7/10

\begin{abstract}
PRINCIPAL COMPONENT ANALYSIS OF NEXAFS SPECTRA FOR MOLYBDENUM SPECIATION IN HYDROTREATING CATALYSTS. Bulk and supported molybdenum based catalysts, modified by nickel, phosphorous or tungsten were studied by NEXAFS spectroscopy at the $\mathrm{Mo}_{\mathrm{III}}$ and $\mathrm{L}_{\text {II }}$ edges. The techniques of principal component analysis (PCA) together with a linear combination analysis (LCA) allowed the detection and quantification of molybdenum atoms in two different coordination states in the oxide form of the catalysts, namely tetrahedral and octahedral coordination.
\end{abstract}

Keywords: XAFS; principal component analysis; hydrotreatment.

\section{INTRODUÇÃO}

Industrialmente, nos processos de hidrotratamento de petróleo, os catalisadores à base de sulfetos de Co-Mo, Ni-W e Ni-Mo, normalmente suportados em $\gamma-\mathrm{Al}_{2} \mathrm{O}_{3}$, ainda são os mais utilizados. Entretanto, o processamento de cargas mais refratárias, principalmente com maior conteúdo de nitrogenados, e a adoção de legislações ambientais mais restritivas têm incentivado a procura por catalisadores mais ativos. Nos últimos 10 anos, surgiram várias pesquisas em torno do uso de aditivos ${ }^{1-3}$ ou de novos suportes ${ }^{2,4}$ visando gerar catalisadores mais ativos. Segundo a literatura, ${ }^{5}$ o tamanho de partícula da fase ativa, a coordenação dos átomos de Mo e sua interação com o suporte têm influência primordial na atividade catalítica.

Embora estes catalisadores sejam empregados na forma sulfetada (sulfetos mistos de Co ou Ni com W ou Mo), sua atividade é consequência direta da natureza e dispersão das espécies presentes nos catalisadores em sua forma oxidada. Estas espécies em geral não são cristalinas e, portanto, não são detectáveis por difração de raios-X (DRX). Por este motivo, neste trabalho, empregou-se a técnica de estrutura fina da absorção de raios-X próxima à borda (NEXAFS ou XANES), que é sensível ao ambiente local dos átomos de interesse, mesmo quando parte de uma estrutura amorfa. ${ }^{6}$

Infelizmente, espectros NEXAFS são, normalmente, de difícil interpretação devido às sutis diferenças apresentadas por diferentes espécies. Por outro lado, na literatura, a técnica de análise por componentes principais (ACP) tem sido de grande utilidade na análise de grandes quantidades de dados em diferentes áreas como, por exemplo, economia, administração, ciências sociais, engenharias, química, biologia etc. $\mathrm{Na}$ área de química existem muitos trabalhos que citam a aplicação desta metodologia. Dentre eles, podemos citar Ferreira et al. ${ }^{7}$ que estudaram os constituintes inorgânicos em sucos e refrigerantes, Wasim et al. ${ }^{8}$ estudaram a desconvolução espectral de espécies e análise de rank em experimentos de cromatografia líquida acoplada com RMN, Silva et al. ${ }^{9}$ aplicaram a ACP na verificação das atribuições de sinais de espectros de $\mathrm{RMN}{ }^{1} \mathrm{H}$ em molécula de 3-aril $(1,2,4)$-oxadiazol-5-carboidrazida benzilidenos.

\footnotetext{
*e-mail: farojr@iq.ufrj.br
}

A própria análise exploratória de espectros NEXAFS também já foi descrita na literatura como, por exemplo, Rumpf et al. ${ }^{10}$ utilizaram a ACP no estudo do mecanismo da reação de troca entre $\mathrm{CuCl}$ e $\mathrm{BaO}$ pela técnica de NEXAFS resolvida no tempo, Fernandéz-García et al. ${ }^{11}$ pesquisaram a influência do estado do metal em catalisadores de três-vias baseados em Pd com NEXAFS in situ e ACP, Jalilehvand et al. ${ }^{12}$ fizeram a caracterização estrutural das espécies de Mo em soluções aquosas de $\mathrm{HCl}$ e Beauchemin et al. ${ }^{13}$ modelaram quantitativamente os espectros NEXAFS na borda $\mathrm{K}$ do enxofre, a partir de amostras de solo húmico.

Wasserman et al. ${ }^{14}$ utilizaram a ACP para acelerar a interpretação de espectros EXAFS. Mostraram que as componentes derivadas dos espectros possuem as mesmas propriedades matemáticas que estes, sendo possível utilizá-las na determinação de comprimentos de ligação e números de coordenação. Assim, o número de componentes a serem analisadas é normalmente inferior ao número de espectros EXAFS.

Por fim, Wang et al. ${ }^{15}$ mostraram que é possível utilizar as técnicas de ACP e de espectroscopia de absorção de raios-X resolvida no tempo para a especiação e determinação da estrutura de intermediários químicos no caso da redução de catalisadores de céria dopados com cobre.

Assim, neste trabalho, esta técnica foi utilizada no sentido de racionalizar os dados de NEXAFS obtidos nas bordas $\mathrm{L}_{\mathrm{III}}$ e $\mathrm{L}_{\mathrm{II}}$ do Mo.

A ACP foi originalmente descrita por Karl Pearson em 1901, ${ }^{16}$ sendo posteriormente consolidada por Hotelling, ${ }^{17} \mathrm{em}$ 1931, com o propósito particular de analisar estruturas de correlações. Aproximadamente 30 anos mais tarde (anos 60), esse tipo de análise foi introduzido na Química por Malinowski. ${ }^{18}$ Como visto, a abordagem da ACP não é nova, mas somente com a difusão nas últimas décadas do uso massivo de computadores está ocorrendo um rápido crescimento em sua utilização.

A análise por componentes principais (ACP) é um método de análise multivariada utilizada para projetar dados n-dimensionais em um espaço de baixa dimensão com o mínimo de perda estatística. Além disso, a análise separa a informação importante da redundante, reconhece padrões de comportamento, detecta amostras que não se encaixam no modelo, resumidamente, facilita a visualização e a interpretação dos resultados. ${ }^{19}$ Isso é feito através do cálculo de 
componentes principais, obtidas como combinações lineares das variáveis originais. A ACP é um método exploratório porque auxilia na elaboração de hipóteses gerais a partir dos dados coletados, contrastando com estudos direcionados nos quais hipóteses prévias são testadas. Em uma análise por componentes principais, o agrupamento das amostras define a estrutura dos dados através de gráficos de scores e loadings, cujos eixos são componentes principais (CP's) nos quais são projetados os dados que serão discutidos adiante. Os scores fornecem a composição das CP's em relação às amostras, enquanto os loadings fornecem essa mesma composição em relação às variáveis. Como as CP's são ortogonais, é possível examinar as relações entre amostras e variáveis através dos gráficos de scores e loadings. A partir da posição espacial dos dados, podem-se calcular as distâncias entre eles e, a partir destas, avaliar as medidas de similaridade e dissimilaridade entre os pontos. Cada ponto pode, assim, ser agrupado aos pontos de maior proximidade espacial, ou pode ser excluído de determinado grupo, dado o seu distanciamento. O estudo conjunto de scores e loadings ainda permite estimar a influência de cada variável em cada amostra..$^{20,21}$

\section{PARTE EXPERIMENTAL}

\section{Catalisadores}

Foram utilizadas duas séries de catalisadores cedidos pelo CENPES-PETROBRAS. Uma série, denominada série MoAlSG, é constituída de catalisadores de $\mathrm{MoO}_{3}-\mathrm{Al}_{2} \mathrm{O}_{3}$ preparados por um método sol-gel, diferindo entre si pela temperatura e $\mathrm{pH}$ durante a síntese dos materiais. A outra série de catalisadores é constituída por catalisadores de molibdênio, mássicos ou suportados em alumina, contendo (ou não) níquel, fósforo e tungstênio. Estes catalisadores foram denominados NiMo e suas composições estão mostradas na Tabela 1.

Tabela 1. Composição dos catalisadores da série NiMo

\begin{tabular}{lccccc}
\hline Amostra & $\mathrm{MoO}_{3}(\%)$ & $\mathrm{NiO}(\%)$ & $\mathrm{P}_{2} \mathrm{O}_{5}(\%)$ & $\mathrm{WO}_{3}(\%)$ & Observação \\
\hline NiMo-1 & 17,6 & 5,6 & 7,8 & - & Suportado \\
NiMo-2 & 21,0 & 31,8 & - & 36,6 & Mássico \\
NiMo-3 & 21,5 & 4,1 & 6,6 & - & Suportado \\
NiMo-4 & 16,5 & 3,4 & - & - & Suportado \\
\hline
\end{tabular}

\section{Análises de absorção de raios-X}

As medidas de XAFS na borda do Mo foram realizadas no Laboratório Nacional de Luz Síncrotron (LNLS), Campinas, na linha D04A-SXS, de raios-X moles, com anel operado a 1,37 GeV de energia e corrente máxima de $250 \mathrm{~mA}$.

Os experimentos foram conduzidos nas bordas $\mathrm{L}_{\mathrm{III}}$ e $\mathrm{L}_{\mathrm{II}}$ do $\mathrm{Mo}$, com energias de, aproximadamente, 2520 e 2620 eV, respectivamente, com detecção feita em modo de rendimento total de elétrons (TEY), utilizando um monocromador de $\mathrm{Si}(111)$. A resolução era em torno de $1,8 \mathrm{eV}$. Todos os espectros foram normalizados após subtração da pré-borda ajustada com uma função linear.

\section{Metodologia da análise por componentes principais}

A ACP é utilizada na decomposição de uma matriz de dados em várias componentes principais independentes e ortogonais. A expressão matemática representando a decomposição em componentes principais da matriz $\mathrm{X}$ pode ser escrita como:

$$
X=U \Theta V^{t}=T V^{t}=\sum_{i=1}^{A} t_{i} t^{t}
$$

$\mathrm{O}$ produto $\mathrm{U} \Theta \mathrm{V}^{\mathrm{t}}$ descreve a decomposição em componentes singulares $u_{i}$ da matriz $X$. As matrizes $U, V$ e $T$ são a matriz de componentes singulares, a matriz de loadings e a matriz de scores, respectivamente. Os vetores coluna $\mathrm{t}_{\mathrm{i}} \mathrm{e}_{\mathrm{i}}(\mathrm{i}=1,2, \ldots, \mathrm{A})$ das matrizes $\mathrm{T}$ e V são chamados vetores de scores e de loadings, respectivamente. A matriz diagonal $\Theta$ coleta os chamados valores singulares, que são iguais à raiz quadrada da variância (autovalores da matriz de covariância, arranjados em ordem decrescente) distribuída sobre cada componente singular. É importante ressaltar a ortogonalidade dos vetores de scores e de loadings (as matrizes $\mathrm{U}^{\mathrm{t}}$.U e $\mathrm{V}^{\mathrm{t}}$. V são matrizes identidade) e o fato que a importância relativa de cada componente singular é decidida pelo autovalor correspondente (variância). O algoritmo de análise por componentes principais utilizado neste trabalho, para MATLAB 7 e baseado na ref. 18, é apresentado na seção Material Suplementar.

\section{RESULTADOS E DISCUSSÃO}

O espectro NEXAFS na borda L do molibdênio é constituído de duas partes, as chamadas bordas $\mathrm{L}_{\mathrm{III}}$ e $\mathrm{L}_{\mathrm{II}}$, que correspondem à absorção de raios-X e ejeção de fotoelétrons dos níveis $2 p_{3 / 2}$ e $2 p_{1 / 2}$, respectivamente (dois níveis diferentes de energia são gerados pelo acoplamento spin-órbita).

As bordas $\mathrm{L}_{\mathrm{III}}$ e $\mathrm{L}_{\mathrm{II}}$ mostram várias diferenças entre si, inclusive em sua forma e na relação entre as intensidades de seus picos constituintes, mostrando ainda um desvio do previsto teoricamente pela teoria do campo cristalino, levando em conta as coordenações observadas. As interações responsáveis pelos desvios observados são efeitos multipletos e o acoplamento spin-órbita nos orbitais $3 \mathrm{~d}$ e 4d..$^{22}$ Além disso, interações eletrônicas e polarizações de spin e dos orbitais dos elétrons de valência alteram a forma e a simetria do estado fundamental, intensificando o desvio observado. No caso de sistemas $4 \mathrm{~d}$ (e.g. Mo, investigado neste trabalho), as bordas $\mathrm{L}_{\mathrm{III}}$ e $\mathrm{L}_{\mathrm{II}}$ são separadas por um largo split, devido ao acoplamento spin-órbita do caroço, sendo este de aproximadamente $100 \mathrm{eV}$. Como nos resultados apresentados a seguir as conclusões obtidas a partir da ACP em ambas as bordas de absorção foram bastante similares, os resultados da borda $\mathrm{L}_{\mathrm{II}}$ não serão mostrados, por questões de espaço.

Neste trabalho foram utilizadas, como padrões estruturais, duas fases oxidadas de molibdênio, o óxido $\mathrm{MoO}_{3}$, onde este se encontra em coordenação octaédrica, e o sal $\mathrm{Na}_{2} \mathrm{MoO}_{4}$, onde este se encontra em coordenação tetraédrica. Como já é conhecido, a análise das tabelas dos grupos de ponto Td e Oh (coordenações tetraédrica e octaédrica, respectivamente) leva à conclusão de que os perfis de desdobramento dos orbitais d para estes grupos são consistentes com dois subníveis, um orbital duplamente degenerado e um triplamente degenerado. No caso da coordenação Td, o nível eletrônico $e$, de menor energia, é duplamente degenerado (referente aos orbitais $\mathrm{d}_{\mathrm{z}}{ }^{2} \mathrm{e}_{\mathrm{x}-\mathrm{y}}{ }^{2}$ ), e o nível eletrônico $t_{2}$, de maior energia, é triplamente degenerado (referentes aos orbitais $\mathrm{d}_{\mathrm{xy}}, \mathrm{d}_{\mathrm{xz}} \mathrm{e} \mathrm{d}_{\mathrm{yz}}$ ). Por outro lado, no caso de coordenação Oh, o nível de menor energia é o triplamente degenerado, $t_{2 g}$, enquanto que o orbital de maior energia é o duplamente degenerado, $e_{g}$. Embora a coordenação local em torno de Mo não seja estritamente Oh e Td nos padrões $\mathrm{MoO}_{3}$ e $\mathrm{Na}_{2} \mathrm{MoO}_{4}$, respectivamente, as distorções em relação a estas coordenações não alteram significativamente as características eletrônicas acima descritas.

Assim, para os grupos de ponto idealizados, os espectros NEXAFS da borda L deveriam ser constituídos de dois picos com áreas proporcionais a 2 e 3 . Dependendo da coordenação, a relação entre os picos é $t_{2}: e=3: 2$, para o tetraédrico e $t_{2 g}: e_{g}=3: 2$ para o octaédrico. A Figura 1 mostra os espectros NEXAFS das bordas $\mathrm{L}_{\mathrm{III}}$, e suas respectivas derivadas segundas (após aplicação de um algoritmo de suavização), para os padrões $\mathrm{MoO}_{3}$ e $\mathrm{Na}_{2} \mathrm{MoO}_{4}$. Observe-se que 
a forma dos espectros experimentais corrobora a discussão acima apresentada sobre as áreas relativas entre os picos, com base na teoria do campo cristalino.
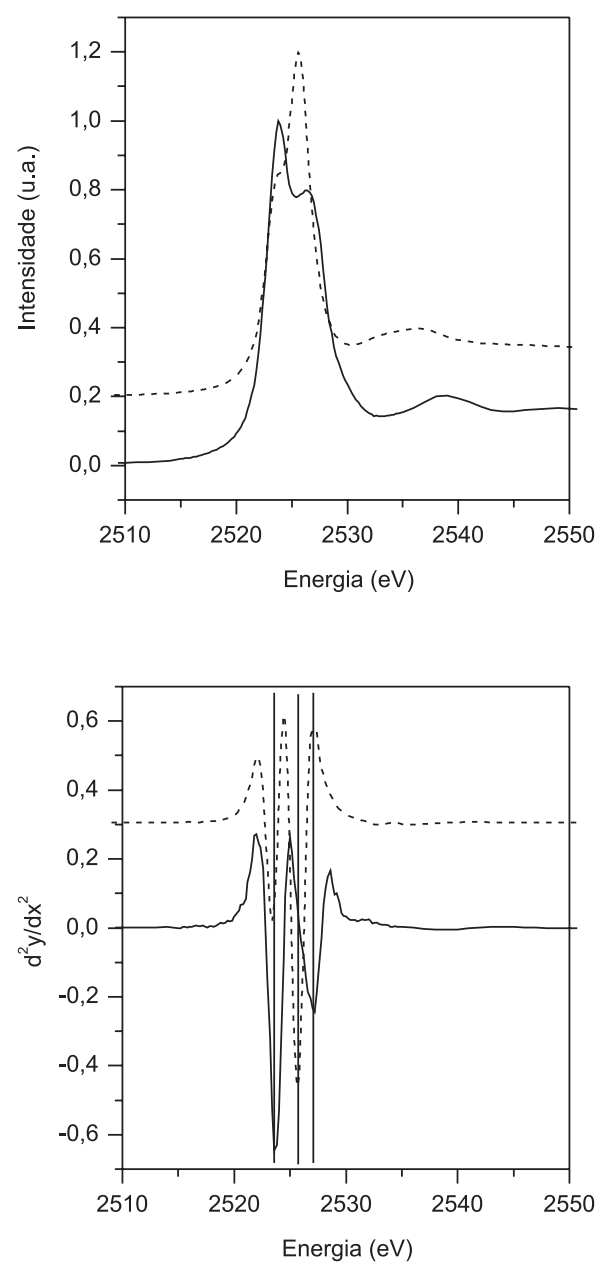

Figura 1. Espectro NEXAFS (esquerda) na borda $L_{I I}$ do Mo e suas respectivas derivadas segundas (direita) para os padrões de $\mathrm{MoO}_{3}$ (Linha) e $\mathrm{Na}_{2} \mathrm{MoO}_{4}$ (Traços)

Na Figura 2a pode ser vista uma comparação entre os espectros NEXAFS da borda $\mathrm{L}_{\mathrm{III}}$ das amostras. Analisando a figura, pode-se perceber a diferença nas intensidades relativas dos picos, melhor evidenciada pela comparação das segundas derivadas dos espectros mostrada na Figura 2b.

Claramente, através da análise das curvas da Figura 2b, vê-se que os espectros das amostras são formados basicamente por três picos principais. O primeiro pico é atribuído à superposição dos picos iniciais referentes aos dois tipos de coordenação, enquanto os dois picos subsequentes, com 2,2 e 3,4 eV (aproximadamente) de separação em relação ao primeiro, são atribuídos aos dois tipos de coordenação nos materiais estudados, tetraédrica e octaédrica, respectivamente. Esta atribuição é corroborada pela distância entre os picos, correspondendo aos desdobramentos dos orbitais $4 \mathrm{~d}$ na análise dos padrões.

Com base nestas conclusões preliminares, partiu-se para o estudo do conjunto de espectros através da técnica de análise por componentes principais.

Para a análise por componentes principais, a matriz de análise, chamada aqui de $\mathbf{y}$, foi construída a partir dos espectros nas bordas $\mathrm{L}_{\text {III }}$ e $\mathrm{L}_{\mathrm{II}}$ (separadamente para cada uma das bordas) das 8 amostras e dos 2 padrões, inseridos como vetores coluna nesta matriz (depois de centrar os dados sobre a média e normalizá-los em relação ao seu desvio padrão). Esta matriz de análise foi decomposta segundo
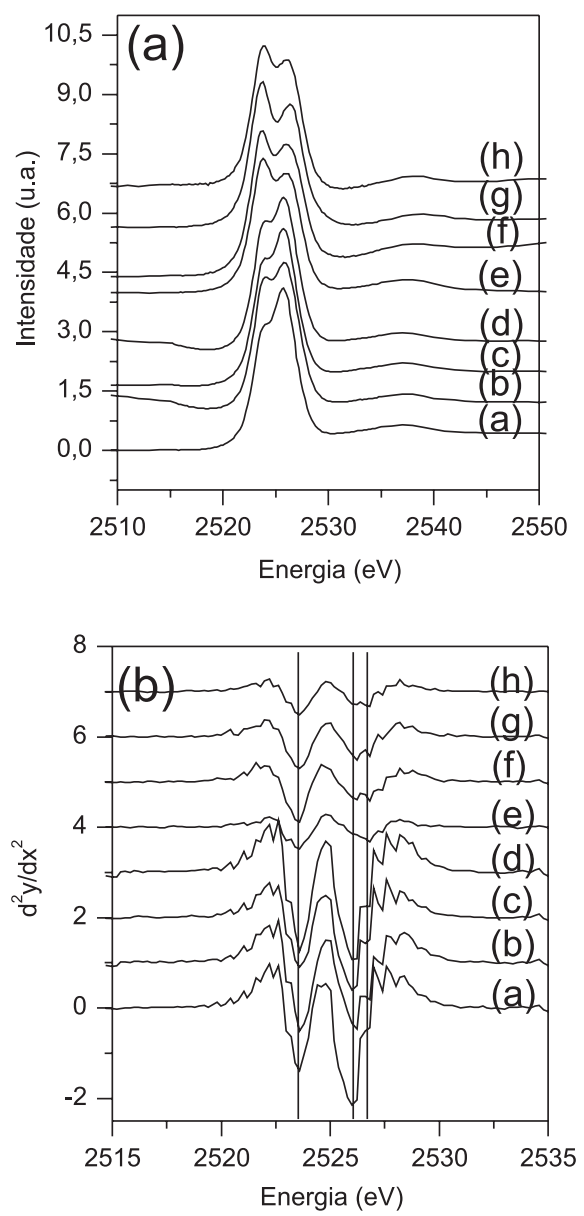

Figura 2. (a) Espectros NEXAFS na borda $L_{I I I}$ do Mo para as diferentes amostras. (b) Derivadas segundas dos espectros NEXAFS: (a) MoAlSG-1, (b) MoAlSG-2, (c) MoAlSG-3, (d) MoAlSG-4, (e) NiMo-1, (f) NiMo-2, (g) NiMo-3 e (h) NiMo-4

o algoritmo de decomposição em componentes singulares, sendo obtidas as matrizes de scores, loadings e autovalores.

Antes de analisar os resultados da ACP aplicada à matriz de espectros NEXAFS, a primeira preocupação é relativa ao número de componentes principais que devem ser retidas na análise, ou seja, quantas componentes se referem a padrões de comportamento exibidos pelos dados e quantas se referem simplesmente a ruído. Um método bastante simples e também bastante utilizado com este intuito é o chamado Scree Test. O Scree Test é um método gráfico inicialmente proposto por R.B. Cattell. ${ }^{23}$ Neste método, locamos as variâncias com um simples gráfico de linha, e o autor sugere achar o ponto onde o decréscimo suave dos autovalores aparentemente se nivela à direita deste ponto. A partir daí, presumivelmente, só poderão ser encontrados Scree fatoriais, onde o termo Scree é utilizado em analogia ao termo geológico que indica os debris que se acumulam na parte inferior de uma inclinação rochosa. De acordo com este critério, em nosso caso, somente serão retidas inicialmente três componentes principais, conforme pode ser observado na Figura 3.

Sabendo quantas componentes principais serão retidas, foram locados os espectros (scores) das três primeiras componentes principais obtidas para a borda $\mathrm{L}_{\mathrm{III}}$, conforme pode ser observado na Figura 4.

Podemos inferir, pela Figura 4, que as componentes principais (gráficos de scores) não são os espectros dos padrões. Isto já era de se esperar, pois, na própria definição do algoritmo de ACP, os eixos sofrem rotação (rotação varimax) de maneira que os novos eixos sejam ortogonais entre si, o que não acontece com os espectros NEXAFS. 


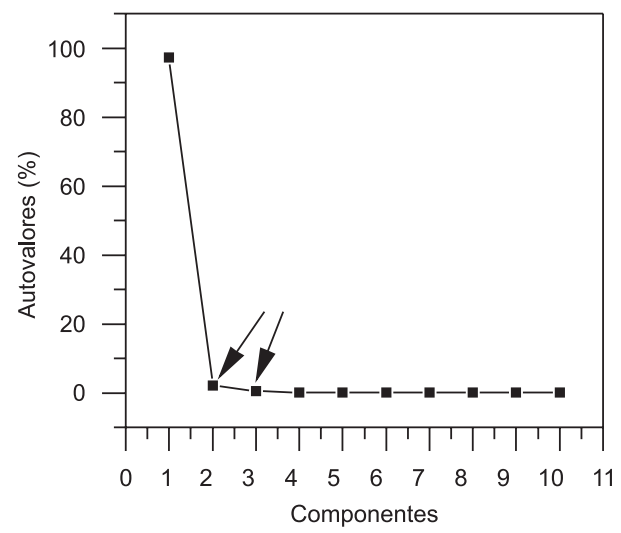

Figura 3. Scree Plot para os autovalores obtidos na análise por componentes principais na borda $L_{I I I}$ do Mo. As setas mostram as possíveis escolhas do número de componentes principais

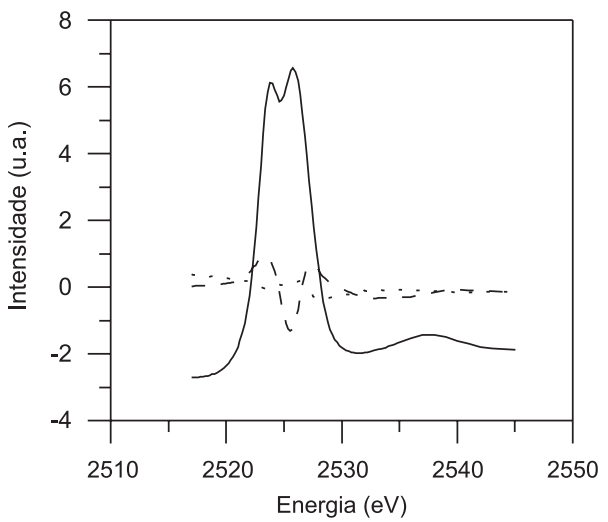

Figura 4. Gráficos de scores relativos às três primeiras componentes principais, representadas por, respectivamente, linha, traços e pontos

É interessante observar que, enquanto os espectros das duas primeiras componentes principais descrevem características marcantes do conjunto de espectros estudados, a terceira componente principal é composta essencialmente de ruído, com alguma influência de desvios de linha de base (provavelmente remanescentes da subtração da pré-borda dos espectros). Isto fez com que esta componente fosse descartada neste trabalho, sendo retidas somente as duas primeiras. Isto significa, essencialmente, que a combinação de apenas dois fatores é capaz de explicar os espectros NEXAFS na borda L do molibdênio de todos os materiais estudados. Sem levar em conta fatores ligados ao preparo dos catalisadores, os materiais diferem entre si em diversos outros, tais como presença ou não de $\mathrm{Ni}, \mathrm{P}, \mathrm{W}$ e $\mathrm{Na}$ (no caso do molibdato de sódio), proporção entre estes elementos, uso ou não de um suporte e tipo de coordenação do molibdênio (tetraédrica ou octaédrica). Por outro lado, têm em comum que todos contêm Mo no estado de oxidação +6 em sua composição. Seguramente todos os demais fatores podem afetar o tipo de coordenação do molibdênio, porém, em princípio, poderiam também influenciar de forma independente o perfil dos espectros NEXAFS. No entanto, a ACP indica que apenas dois fatores independentes são responsáveis por $99,4 \%$ da variância dos espectros.

Na Figura 5 estão locados os valores de $\mathrm{CP} 2$ x CP1, como é comum na representação de resultados de ACP. Nesta figura, podemos observar que as projeções dos espectros NEXAFS da borda $\mathrm{L}_{\mathrm{III}}$ do Mo nos novos eixos ortogonais, referentes às duas primeiras componentes principais, são praticamente invariáveis para as diferentes amostras no caso da CP1 (Componente Principal 1) e apresentam diferenças marcantes no caso da CP2 (Componente Principal 2). Este fato é espe- rado, tendo em vista que a primeira componente principal representa 97,3\% da variância total do sistema, ou seja, revela um componente espectral que está presente em todas as amostras (a forma geral dos espectros). Já quando consideramos a segunda componente principal, representando $2,1 \%$ da variância do sistema, podem ser observados os detalhes dos espectros, ou seja, as pequenas diferenças entre os espectros das diferentes amostras.

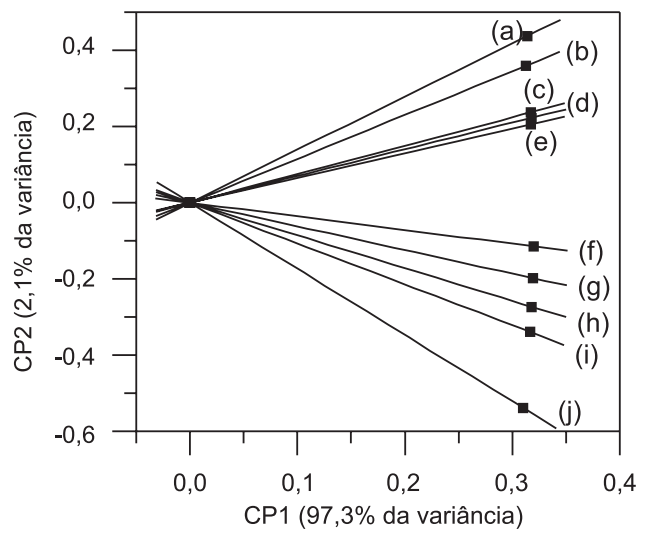

Figura 5. Gráfico de loadings para as duas primeiras componentes principais CP2 2 CP1, que representam $99,4 \%$ da variância total do sistema. (a) $\mathrm{MoO}_{3}$, (b) NiMo-3, (c) NiMo-4, (d) NiMo-1, (e) NiMo-2, (f) MoAlSG-2, ( $g$ ) MoAlSG-4, (h) MoAlSG-3, (i) MoAlSG-1, (j) $\mathrm{Na}_{2} \mathrm{MoO}_{4}$

Este tipo de gráfico obtido na Figura 5, onde as amostras se apresentam distribuídas radialmente ao redor de um eixo, já é bastante conhecido para este método e, normalmente, representa sistemas a dois componentes, onde as diferentes amostras possuem diferentes proporções destes. Ainda observando esta mesma figura, vemos que os compostos de molibdênio, $\mathrm{MoO}_{3}$ e $\mathrm{Na}_{2} \mathrm{MoO}_{4}$, apresentam-se como extremos em CP2. O que estes compostos têm em comum é o fato de terem molibdênio em estado de oxidação +6 coordenado a oxigênio. Este deve ser associado à componente principal $\mathrm{CP} 1$, que é praticamente invariante entre as diferentes amostras. Diferem quanto ao estado de coordenação do molibdênio e quanto à presença de sódio. No entanto, o teor de sódio não pode ser a CP2, pois, se assim fosse, sua contribuição seria nula para os espectros dos catalisadores, o que não foi o caso. Assim, CP2 deve ser associada ao estado de coordenação do molibdênio, tetraédrico ou octaédrico.

A partir deste ponto, podemos quantificar as proporções dos dois componentes de duas maneiras distintas: na primeira, estimam-se os teores de cada componente no gráfico de $\mathrm{CP} 2 \times \mathrm{CP} 1$ a partir do ângulo entre os vetores que ligam os pontos $[0,0]$ e $[\mathrm{CP} 2, \mathrm{CP} 1]$ para amostra e padrões; a segunda forma trata da análise por combinação linear (ACL), onde os espectros das diferentes amostras são representados por combinações lineares dos espectros de padrões previamente escolhidos. $^{24}$

Neste método forma-se uma matriz problema $\mathbf{y}$ (onde as colunas representam os espectros das amostras) e uma matriz de padrões $\mathbf{x}$ (correspondendo aos espectros dos padrões $\mathrm{MoO}_{3}$ e $\mathrm{Na}_{2} \mathrm{MoO}_{4}$ ). A partir destas matrizes, pela aplicação do método dos mínimos quadrados, obtém-se uma matriz de coeficientes $\mathbf{a}$.

A matriz de coeficientes, depois de normalizada e multiplicada por cem, fornece as porcentagens relativas dos dois componentes em todas as amostras. É interessante ressaltar que a matriz de coeficientes só seria obtida já normalizada se todos os "vetores espectros" possuíssem normas idênticas ,como previsto pela álgebra linear.

Em ambos os casos, a qualidade das simulações foi verificada através do valor de $\mathrm{R}^{2}$ em unidades percentuais, como definido abaixo. ${ }^{25}$ 


$$
R^{2}=100 \cdot\left(1-\frac{\left\|\mathbf{y y}-_{\text {rec }}\right\|^{2}}{\|\mathbf{y}\|^{2}}\right)
$$

onde $\mathbf{y}_{\text {rec }}$ refere-se à matriz de dados $\mathbf{y}$ simulada pelas técnicas.

A Tabela 2 mostra os resultados obtidos pelos dois métodos (ângulos e análise por combinação linear) em ambas as bordas ( $\mathrm{L}_{\mathrm{III}}$ e $\mathrm{L}_{\mathrm{HI}}$ ), junto com os respectivos valores de $\mathrm{R}^{2}$ obtidos com base nos espectros recuperados em cada um dos casos (utilizando duas componentes para a ACP no caso do método dos ângulos ou usando a relação $\mathbf{y}_{\text {rec }}=\mathbf{x} . \mathbf{a}$, no caso da análise por combinação linear).

Tabela 2. Resultados de percentual de Mo tetraédrico nas diferentes amostras como determinado pelos métodos dos ângulos e análise por combinação linear (ACL) nas duas bordas de absorção de raios-X. Na última linha são mostrados os resultados do parâmetro $\mathrm{R}^{2}$

\begin{tabular}{|c|c|c|c|c|}
\hline AmostralMétodo & Ângulos- $\mathrm{L}_{\text {III }}$ & ACL-L ${ }_{\text {III }}$ & Ângulos- $\mathrm{L}_{\mathrm{II}}$ & ACL-L \\
\hline NiMo-3 & 4,9 & 4,3 & 2,1 & 4,0 \\
\hline NiMo-4 & 11,2 & 22,3 & 14,1 & 20,4 \\
\hline NiMo-1 & 12,8 & 25,9 & 18,9 & 26,1 \\
\hline NiMo-2 & 14,7 & 27,1 & 19,2 & 28,1 \\
\hline MoAlSG-2 & 63,0 & 55,6 & 82,8 & 62,3 \\
\hline MoAlSG-4 & 74,1 & 64,2 & 92,0 & 70,6 \\
\hline MoAlSG-3 & 82,2 & 71,7 & 97,3 & 78,9 \\
\hline MoAlSG-1 & 88,0 & 77,9 & 99,1 & 82,6 \\
\hline $\mathrm{R}^{2}$ & 99,90 & 97,78 & 99,87 & 97,63 \\
\hline
\end{tabular}

Observando os valores obtidos para $\mathrm{R}^{2}$ (Tabela 2), pode-se inferir a boa qualidade das simulações, indicando que a escolha de reter duas componentes principais conforme indicado pelo Scree Test é adequada a este sistema que está sendo analisado. A Figura 6 mostra uma comparação entre os espectros NEXAFS experimentais e recuperados através dos métodos ACP e ACL, no caso da amostra MoAlSG-1. Verifica-se que ambos os métodos recuperam adequadamente os espectros experimentais, como já havia sido sugerido pelos valores de $\mathrm{R}^{2}$ mostrados na Tabela 2.

Através dos resultados indicados na Tabela 2, observa-se que as análises por ambos os métodos levam a resultados qualitativamente concordantes em relação aos teores de Mo em coordenação tetraédrica. Por outro lado, no caso do método dos ângulos observa-se grande discrepância (dispersão em torno de 15\%) entre os resultados obtidos utilizando as bordas $\mathrm{L}_{\mathrm{II}}$ e $\mathrm{L}_{\mathrm{III}}$, enquanto que o método de análise por combinação linear gera resultados bastante semelhantes em ambas as bordas de absorção (dispersão em torno de 4\%), mostrando assim sua maior confiabilidade. Esta discrepância observada entre os resultados obtidos nas bordas $\mathrm{L}_{\mathrm{II}}$ e $\mathrm{L}_{\mathrm{III}}$ no caso do método dos ângulos indica que o mesmo não é adequado para medidas quantitativas neste sistema, porém, a utilização das informações obtidas através da ACP no método de análise por combinação linear (ACL) produziu resultados quantitativos satisfatórios. É importante mencionar que a ACP possui a vantagem de não necessitar, a priori, da utilização de padrões para serem obtidas informações sobre o sistema em estudo como, por exemplo, determinar o número de espécies que representam o conjunto de amostras analisadas, enquanto que no método ACL o número de componentes e suas identidades devem ser especificados para sua execução (informação passível de ser obtida através do método ACP).

Os resultados obtidos mostram que os catalisadores que contêm Ni e Mo são compostos predominantemente por molibdênio octaédrico, principalmente no caso da amostra NiMo-3 (catalisador com fósforo e maior teor de molibdênio), em que praticamente todos os átomos de molibdênio (96\%) se encontram nesta coordenação. No caso das amostras da série MoAlSG, os átomos de molibdênio se
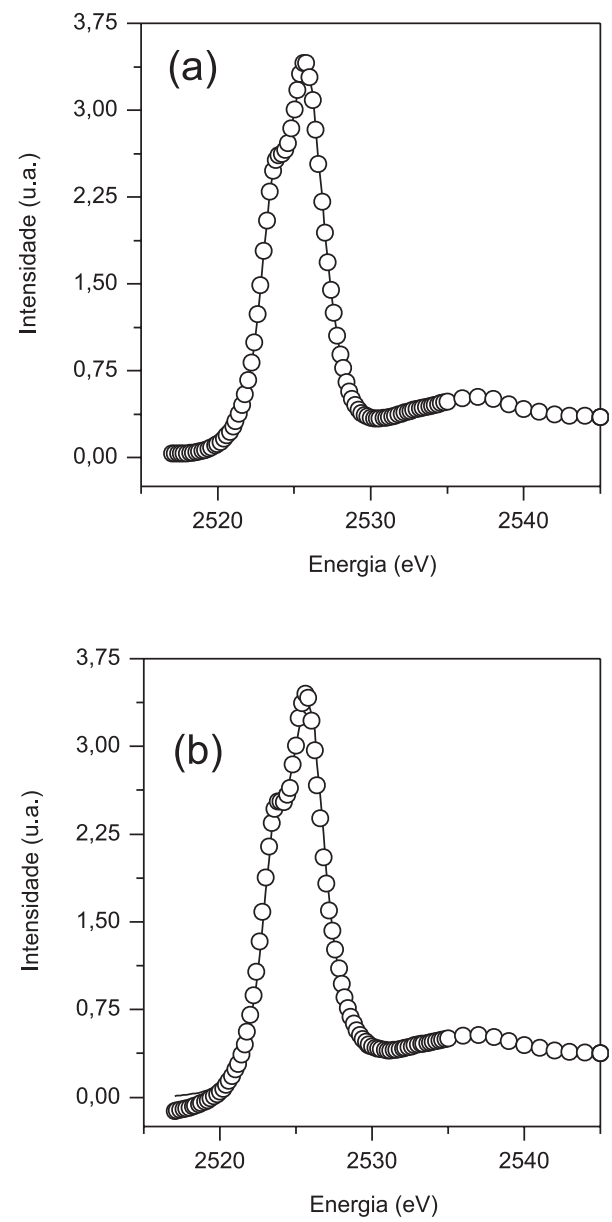

Figura 6. Comparação entre os espectros NEXAFS experimental e recuperado através dos métodos ACP (a) e ACL (b) para a amostra MoAlSG-1. (-) espectros experimentais, ( $($ ) espectros recuperados

encontram predominantemente em coordenação tetraédrica, com pequena variação entre os resultados para as diferentes amostras (dispersão de aproximadamente $10 \%$ em relação à média das amostras nesta série).

\section{CONCLUSÃO}

A análise por componentes principais mostrou-se uma técnica rápida e eficaz na análise de espectros NEXAFS, permitindo determinar o número de fatores independentes que determinam o espectro de uma série de amostras relacionadas entre si, bem como a contribuição relativa destes fatores para os espectros. A inclusão de padrões de estrutura e composição conhecidas na série de amostras permitiu associar as componentes principais a fatores com significado químico e quantificar estes fatores nas diferentes amostras.

Assim, numa série de óxidos mistos de molibdênio com um ou mais de outros elementos (Ni, W, Al e P), com diferentes proporções entre os elementos constituintes e preparados por distintos métodos, foi possível identificar que apenas um fator é capaz de explicar as diferenças entre os espectros NEXAFS dos materiais nas bordas $\mathrm{L}_{\mathrm{II}}$ e $\mathrm{L}_{\text {III }}$ do molibdênio, sendo este fator o estado de coordenação do molibdênio nas amostras, já que em todas elas o molibdênio estava no estado de oxidação +6 .

Foi então possível, através do método ACL, determinar a proporção do molibdênio em cada estado de coordenação nas amostras, por combinação linear entre os espectros NEXAFS de padrões representativos do molibdênio em cada um deles. 
Esta especiação do molibdênio entre diferentes estados de coordenação, que foi independente da escolha da borda de absorção do molibdênio $\left(\mathrm{L}_{\mathrm{II}}\right.$ ou $\left.\mathrm{L}_{\mathrm{III}}\right)$, é de grande importância no processo de pesquisa e desenvolvimento de novos catalisadores de hidrotratamento e pode ser efetuada de forma mais efetiva do que é possível através de outras técnicas, tais como as espectroscopias vibracionais (Raman e IV) e eletrônica.

\section{MATERIAL SUPLEMENTAR}

Está disponível em http://quimicanova.sbq.org.br, na forma de arquivo .PDF, com acesso livre.

\section{AGRADECIMENTOS}

À S. S. X. Chiaro e a J. L. Zotin pelas amostras dos catalisadores de molibdênio. Ao CENPES-PETROBRAS por financiar este trabalho e ao "Laboratório Nacional de Luz Síncrotron" pela aprovação do projeto e pelo auxílio financeiro para as medidas NEXAFS na borda L do Mo. J.-G. Eon agradece ao CNPq pelo suporte financeiro concedido durante a realização do projeto. V. O. Rodrigues agradece à CAPES pela bolsa de doutorado.

\section{REFERÊNCIAS}

1. Ramírez, J.; Gutiérrez-Alejandre, A.; Langmuir 1998, 14, 630.

2. Santes, V.; Díaz, L.; Appl. Catal., A 2005, 281, 121; Saih, Y.; Segawa, K.; Catal. Today 2003, 86, 61; Damyanova, S.; Jiratova, K.; Appl. Catal., A 1995, 125, 257.

3. Öhman, L. O.; Paul, J.; Mater. Chem. Phys. 2002, 73, 242.

4. Sivakumar, S.; Mater. Lett. 2004, 58, 2664; Kaneko, E. Y.; da Silva, V. T.; Appl. Catal., A 2002, 235, 71; Ramírez, J.; Gutiérrez-Alejandre, A.; Microporous Mesoporous Mater. 1998, 23, 265; Montoya, J. A.; J. Mater. Chem. 2001, 11, 944; Maity, S. K.; Appl. Catal., A 2003, 244, 141; Ramírez, J.; Gutiérrez-Alejandre, A.; J. Catal. 1997, 170, 108; Borque, M. P.; Ramírez, J.; Appl. Catal., A 1999, 180, 53; Linacero, R.; RojasCervantes, M. L.; J. Mater. Sci. 2000, 35, 3279.
5. Ramírez, J.; Catal. Today 2004, 98, 19

6. Rehr, J. J.; Albers, R. C.; Rev. Mod. Phys. 2000, 72, 621.

7. Ferreira, E. C.; Rodrigues, S. H. B. G.; Ferreira, M. M. C.; Nóbrega, J. A.; Nogueira, A. R. A.; Eclética Química 2002, 27, 77.

8. Wasim, M.; Brereto, R. G.; Chem. Int. Lab. Syst. 2004, 72, 133.

9. da Silva, J. B. P.; Malvestiti, I.; Hallwas, F.; Ramos, M. N.; Leite, L. F. C. C.; Barreiro, E. J.; Quim. Nova 2005, 28, 492.

10. Rumpf, H.; Janssen, J.; Modrow, H.; Winkler, K.; Hormes, J.; J. Solid State Chem. 2002, 163, 158.

11. Fernández-García, M.; Iglesias-Juez, A.; Martínez-Arias, A.; Hungría, A. B.; Anderson, J. A.; Conesa, J. C.; Soria, J.; J. Catal. 2004, 221, 594.

12. Jalilehvand, F.; Mah, V.; Leung, B. O.; Ross, D.; Parvez, M.; Aroca, R. F.; Inorg. Chem. 2007, 46, 4430.

13. Beauchemin, S.; Hesterberg, D.; Beauchemin, M.; Soil Sci. Soc. Am. J. 2002, 66, 83 .

14. Wasserman, S. R.; Allen, P. G.; Shuh, D. K.; Bucher, J. J.; Edelstein, N. M.; J. Synchrotron Rad. 1999, 6, 284.

15. Wang, Q.; Hanson, J. C.; Frenkel, A. I.; J. Chem. Phys. 2008, 129, 234502.

16. Pearson, K.; Phil. Mag. J. 1901, 6, 559.

17. Hotelling, H.; J. Educ. Psychol. 1931, 24, 417.

18. Malinowski, E. R.; Factor Analysis in Chemistry, $3^{\text {rd }}$ ed., Wiley: New York, 2002.

19. Moita Neto, J. M.; Moita, G. C.; Quim. Nova 1998, 21, 467; Egreja Filho, F. B.; Reis, E. L.; Jordão, C. P.; Pereira Neto, J. T.; Quim. Nova 1999, 22, 324

20. Ferreira, M. M. C.; Antunes, A. M.; Melgo, M. S.; Volpe, P. L. O.; Quim. Nova 1999, 22, 724.

21. Sena, M. M.; Poppi, R. J.; Frighetto, R. T. S.; Valarini, P. J.; Quim. Nova 2000, 23, 547; Haswell, S. J.; Walmsley, A. D.; J. Anal. At. Spectrom. 1998, 13, 131; Blanco-Gomis, D.; Fernandez-Rubio, P.; GutiérrezAlvarez, M. D.; Mangas-Alonso, J. J.; Analyst 1998, 123, 125.

22. de Groot, F. M. F.; Physica B 1995, 208-209, 15.

23. Cattel, R. B.; Multivariate Behav. Res. 1966, 1, 245.

24. Lengke, M. F.; Ravel, B.; Fleet, M. E.; Wanger, G.; Gordon, R. A.; Southam, G.; Environ. Sci. Technol. 2006, 40, 6304.

25. Tauler, R.; Anal. Chim. Acta 2007, 595, 289. 


\section{ANÁLISE POR COMPONENTES PRINCIPAIS DE ESPECTROS NEXAFS NA ESPECIAÇÃO DO MOLIBDÊNIO EM CATALISADORES DE HIDROTRATAMENTO}

\section{Arnaldo da C. Faro Jr.*, Victor de O. Rodrigues e Jean-G. Eon}

Instituto de Química, Universidade Federal do Rio de Janeiro, Centro de Tecnologia, Bl. A, Cidade Universitária, $21949-900$ Rio de Janeiro - RJ, Brasil

\section{Angela S. Rocha}

COPPE - Instituto Alberto Luiz Coimbra de Pós-graduação e Pesquisa de Engenharia, Universidade Federal do Rio de Janeiro, CP 68502, Cidade Universitária, 21949-900 Rio de Janeiro - RJ, Brasil

O algoritmo de ACP para Matlab 7 mostrado a seguir necessita de dois conjuntos de dados de entrada: a matriz ACP, formada pelas absorções dos espectros de NEXAFS inseridos como colunas e um vetor coluna contendo as energias dos espectros, chamado de vetor Energia.

$$
\begin{gathered}
{[\mathrm{n}, \mathrm{m}]=\text { size }(\mathrm{ACP}) ;} \\
\text { 'Centrando os dados na média: } \\
\text { média = mean }(\mathrm{ACP}) ; \\
\text { acp_centrada=ACP-ones }(\mathrm{n}, 1) * \text { média; }
\end{gathered}
$$

'Normalizando os dados em relação ao desvio padrão:

$$
\text { acp_norm = std (ACP); }
$$

$\mathrm{X}=$ acp_centrada.$/($ ones $(\mathrm{n}, 1) *$ acp_norm);

,Decomposição em valores singulares da matriz de trabalho X:

$[\mathrm{u}$ Theta $\mathrm{v}]=\operatorname{svd}(\mathrm{X})$;

'Obtendo a matriz de variâncias e grafando o Scree Plot: variância $=\operatorname{diag}($ Theta $) . \wedge 2$;

Var_percentual = variância/sum $($ variância $) * 100$; plot(Var_percentual);

'Obtendo as matrizes de scores, loadings e autovalores:

$$
\begin{gathered}
\text { loadings }=\mathrm{v} ; \\
\text { eigenvalues }=\mathrm{Theta} ; \\
\text { scores }=\mathrm{u} * \text { Theta; }
\end{gathered}
$$

'Grafando os tres primeiros vetores de scores e as diferentes com-

ponentes principais:

$$
\text { figure; }
$$

$\operatorname{subplot}(2,2,1) ;$ plot (Energia, scores(:,1),'ro', Energia, scores(:,2),'bo', Energia, scores(:,3),'go');

subplot (2,2,2);plot (loadings(:,1), loadings (:,2),'o'); subplot (2,2,3);plot (loadings (:,1), loadings (:,3),'o'); subplot (2,2,4);plot (loadings (:,2), loadings (:,3),'o');

'Recuperando a matriz de dados originais retendo duas componen-

$$
\begin{gathered}
\text { tes principais: } \\
\mathrm{d}=\operatorname{scores}(:, 1: 2) ; \\
\mathrm{e}=\operatorname{loadings}(:, 1: 2) ; \\
\text { X_rec= }=\mathrm{d} * \mathrm{e} ;
\end{gathered}
$$

acp_centrada_rec $=X \_r e c . *(o n e s(n, 1) *$ acp_norm $)$;

ACP_rec=acp_centrada_rec+ones $(n, 1) *$ média;

'Calculando os resíduos e o valor de $\mathrm{R}^{2}$ : resíduos $=\mathrm{ACP}-\mathrm{ACP} \_$rec;

$\mathrm{R} 2=100 *\left(1-\left(\right.\right.$ norm $\left.\left.(\text { residuos,'fro' })^{\wedge} 2 / \operatorname{norm}\left(\mathrm{ACP}, \text { fro' }^{\wedge}\right)^{\wedge} 2\right)\right)$

*e-mail: farojr@iq.ufrj.br 